\title{
Blood-Brain Barrier Disturbances and Potential Complications of Endovascular Management in Stroke-Technical Note with Limited Review
}

\author{
Sharad B. Ghatge ${ }^{1}$ Pratik S. Itti ${ }^{2, \odot ~ A n j a l i ~ P ~ D e s h m u k h ~}{ }^{3}$
}

\footnotetext{
${ }^{1}$ Department of Radiology and Imaging, Division of Interventional Radiology, Grant Government Medical College and Sir JJ Group of Hospitals, Mumbai, Maharashtra, India

2Department of Radiology and Imaging, Grant Government Medical College and Sir JJ Group of Hospitals, Mumbai, Maharashtra, India

${ }^{3}$ Department of Radiology, Bombay Hospital, Mumbai,

Maharashtra, India
}

\begin{abstract}
Address for correspondence Pratik S. Itti, MBBS, Department of Radiology and Imaging, Grant Government Medical College and Sir JJ Group of Hospitals, Mumbai, Maharashtra, 400008, India (e-mail: ittipratik@gmail.com).
\end{abstract}

\section{Abstract}

Keywords

- BBB disturbances

- contrast enhancement

- contrast extravasation

- hemorrhagic transformation

- cerebral hyperperfusion syndrome
Contrast enhancement (CE), contrast extravasation (CX), hemorrhagic transformation (HT), and cerebral hyperperfusion syndrome (CHS) in patients who have suffered ischemic stroke and have undergone revascularization. There are a handful of articles addressing these pathologies separately. But there is scant literature available combining them together, as the underlying pathophysiology involves disturbances of bloodbrain barrier (BBB). We have reviewed literature and proposed a common mechanism for these events. We systematically searched PubMed, LibGen, Cochrane, and Sci-Hub databases for the studies published online regarding CE, CX, HT, and CHS after endovascular treatment for stroke. This review was conducted based on the PRISMA guidelines. The following medical search terms were used for the online search: contrast enhancement, contrast extravasation, hemorrhagic transformation, cerebral hyperperfusion syndrome, endovascular treatment, contrast staining, postprocedural attenuation, carotid stenting, intra-arterial thrombolysis, and stroke. We did a limited review of literature by analyzing the relevant articles and research papers published to date. We have randomly included prototype cases of $\mathrm{CE}, \mathrm{CX}, \mathrm{HT}$, and $\mathrm{CHS}$ which we have encountered in our Interventional Department from our own database. In compliance with PRISMA guidelines, we screened 33 articles dealing with CE, 32 with CX, 26 articles that addressed $C E$ and $C X$ both, 53 articles dealing with $H T$, and 42 articles dealing with CHS. Overall, 88 articles were filtered on studying the abstract. Further, 15 more had to be excluded as reasoned in the flowchart, and finally 71 articles were included in our study, as again shown in the flowchart. We studied and discussed these articles and research papers in relation to pathophysiology, predisposing factors, preventive measures, and current treatment protocols. BBB disruption is the primary event in $\mathrm{CE}$,
DOI https://doi.org/ $10.1055 / \mathrm{s}-0041-1726167$ ISSN 2457-0214.
(C)2020. Indian Society of Vascular and Interventional Radiology. This is an open access article published by Thieme under the terms of the Creative Commons Attribution-NonDerivative-NonCommercial-License, permitting copying and reproduction so long as the original work is given appropriate credit. Contents may not be used for commercial purposes, or adapted, remixed, transformed or built upon. (https://creativecommons.org/licenses/by-nc-nd/4.0/).

Thieme Medical and Scientific Publishers Pvt. Ltd. A-12, 2nd Floor, Sector 2, Noida-201301 UP, India 
$\mathrm{CX}, \mathrm{HT}$, and CHS with varying severity. Minimizing dose of contrast, optimum timing of revascularization and dose of thrombolytic, judicious selection of mechanical thrombectomy cases, and strict control of blood pressure in postrevascularization period are recommended preventive measures. High-index of clinical suspicion, early imaging to detect, and following-up the same on sequential imaging are key to avoid severe forms of $\mathrm{HT}$ and $\mathrm{CHS}$.

\section{Introduction}

Brain and spinal cord have special barrier for the molecules in blood to be selectively transmitted into the extracellular space around their neurons. This is called the blood-brain barrier (BBB). One of the best ways to know the permeability of BBB on cross-sectional imaging involves injection of contrast agents. Administration of the contrast material intravascularly or via cerebrospinal fluid (CSF) spaces was known since the mid-1970s with regard to CT scan and the mid-1980s with respect to MRI. ${ }^{1}$ During our practice in intervention neuroradiology (IR), we have seen cases of contrast enhancement (CE), contrast extravasation (CX), hemorrhagic transformation (HT), and cerebral hyperperfusion syndrome (CHS) in patients who have suffered ischemic stroke and have undergone revascularization. There are handful of articles addressing these pathologies separately. But there is scant literature available combining them together, as the underlying pathophysiology involves disturbances of BBB. We have reviewed literature and proposed a common mechanism for these events.

\section{Study Design}

\section{Acquisition of Data \\ Data Source}

We systematically searched PubMed, LibGen, Cochrane, and Sci-Hub databases for the studies published online regarding CE, CX, HT, and CHS after endovascular treatment for stroke. This review was conducted based on the PRISMA guidelines. The following medical search terms were used for the online search: contrast enhancement, contrast extravasation, hemorrhagic transformation, cerebral hyperperfusion syndrome, endovascular treatment, contrast staining, post procedural attenuation, carotid stenting, intra-arterial thrombolysis, and stroke (-Fig. 1).

\section{Selection Criteria}

Studies included in the systematic review of literature met the following criteria:

1. Studies demonstrating $C E$ and $C X$ in patients with acute stroke.

2. Studies involving the risk of HT after endovascular therapy for stroke.

3. Studies describing CHS after carotid stenting.
Exclusion criteria included:

1. Types of publications other than clinical studies and research papers, such as letters, editorials and case reports/series ( $<10$ patients).

2. Studies considering carotid endarterectomy as a treatment modality in articles dealing with CHS.

\section{Analysis of Data}

We did a limited review of literature by analyzing the relevant articles and research papers published to date. We randomly included prototype cases of $\mathrm{CE}, \mathrm{CX}, \mathrm{HT}$, and CHS which we encountered in our Interventional Department from our own database. In compliance with PRISMA guidelines, we screened 33 articles dealing with CE, 32 with CX and 26 articles that addressed CE and CX both, 53 articles dealing with HT, and 42 articles dealing with CHS. Overall, 88 articles were filtered on studying the abstract. Further, 15 more had to be excluded as reasoned in the flowchart, and finally 71 articles were included in our study, as again shown in the flowchart. We studied and discussed these articles and research papers in relation to pathophysiology, predisposing factors, preventive measures, and current treatment protocols.

\section{Discussion}

Anatomy and pathophysiology of the BBB: The BBB is a specialized structure consisting of endothelial cells, pericytes, astrocytes, microglia and neuronal cells along with the extracellular matrix, making it a neurovascular unit. The endothelial cells of BBB have tight junctions between them devoid of any fenestrations, with potential ability of pinocytosis. It has unique, selective capability of enzymatic degradation of unwanted and therapeutic molecules. They have abundant mitochondria which are the powerhouse of energy and nutrition. The basement membrane of microvessels is embedded with pericytes, which are vascular smooth muscle cells. The pericytes and endothelial cells are surrounded by basal lamina, which is continuous with astrocytic processes. Astrocytes provide support and maintain integrity of the BBB. The basal lamina acts as an interface for intercellular activities. The moment ischemia sets in, two-way communication between neurons and microvessels is disrupted. Microglial cells are scavenger cells with phagocytic potential mediated by cytokines, inflammatory markers, and 


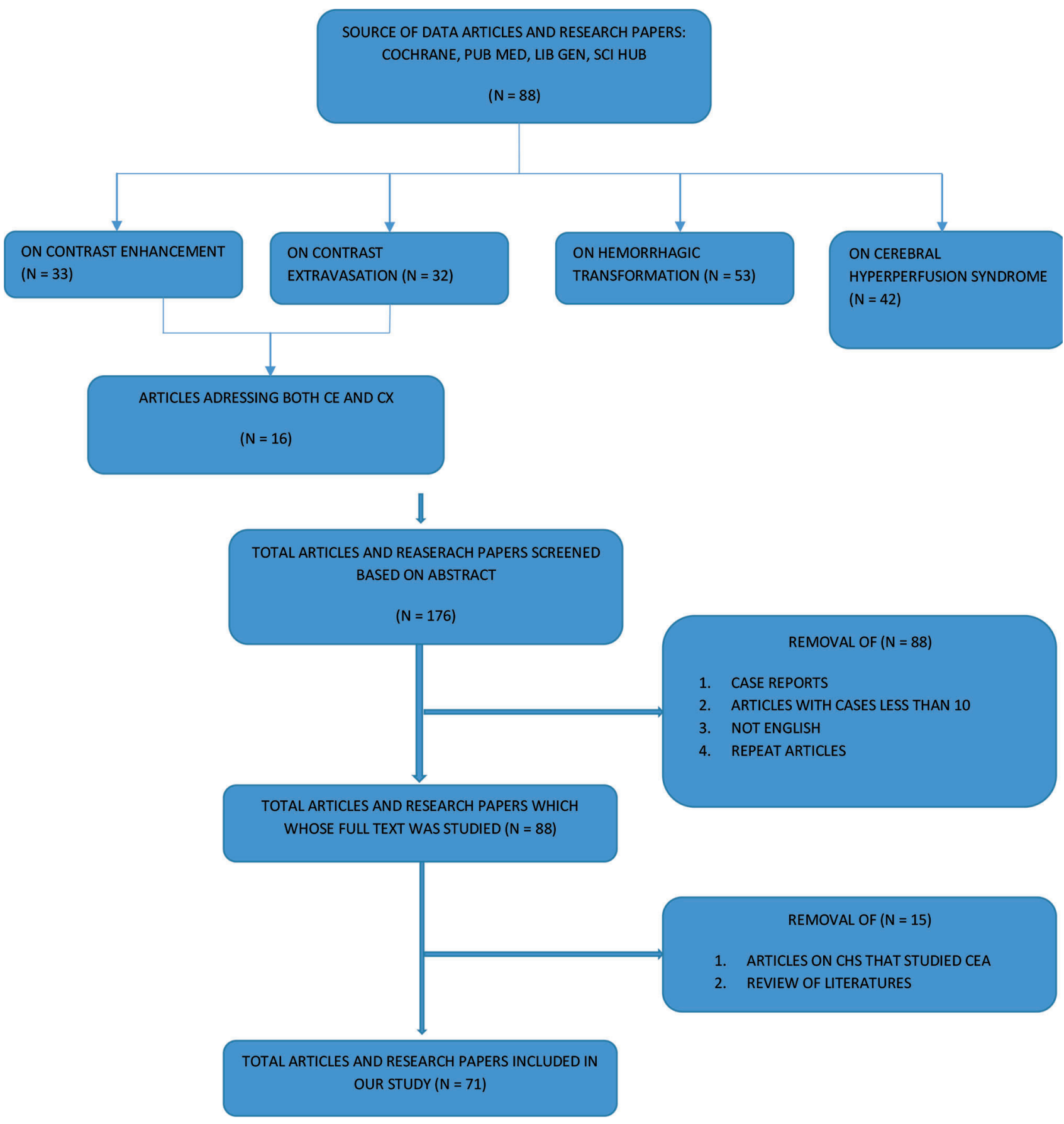

Fig. 1 Flowchart showing study design.

immunomodulation. Ischemic insult activates microglial cells and prepares them for phagocytic activities. One major impact of the decreased cerebral blood flow is state of energy deficiency, with resultant rise in lactic acid and intracellular potassium and release of glutamate, which initiates the BBB disruption. BBB disruption can be in the form of increased permeability, with pinocytosis or more advanced tight junction abnormalities accompanied by diapedesis. Reperfusion results in free radical injury, causing more severe disruption. Thrombolytic agents too have adverse effect on BBB after median time of 3.8 hours, implying that we must finish thrombolytic therapy earlier than this to avoid hemorrhagic transformation. Authors have personal experience about these events only in interventional procedures. We have our own cases to be shown from interventional procedure. We did not have any experience or cases to be shown from other revascularization procedures; hence, carotid endarterectomy (CEA) articles were excluded from our study. A compounding factor leading to CHS in CEA is different and not associated with interventional procedures like denervation of baroreceptors in CEA as against baroceptor stimulation in carotid stenting (CAS) (- Figs. 2 and $\mathbf{3}$ ). 


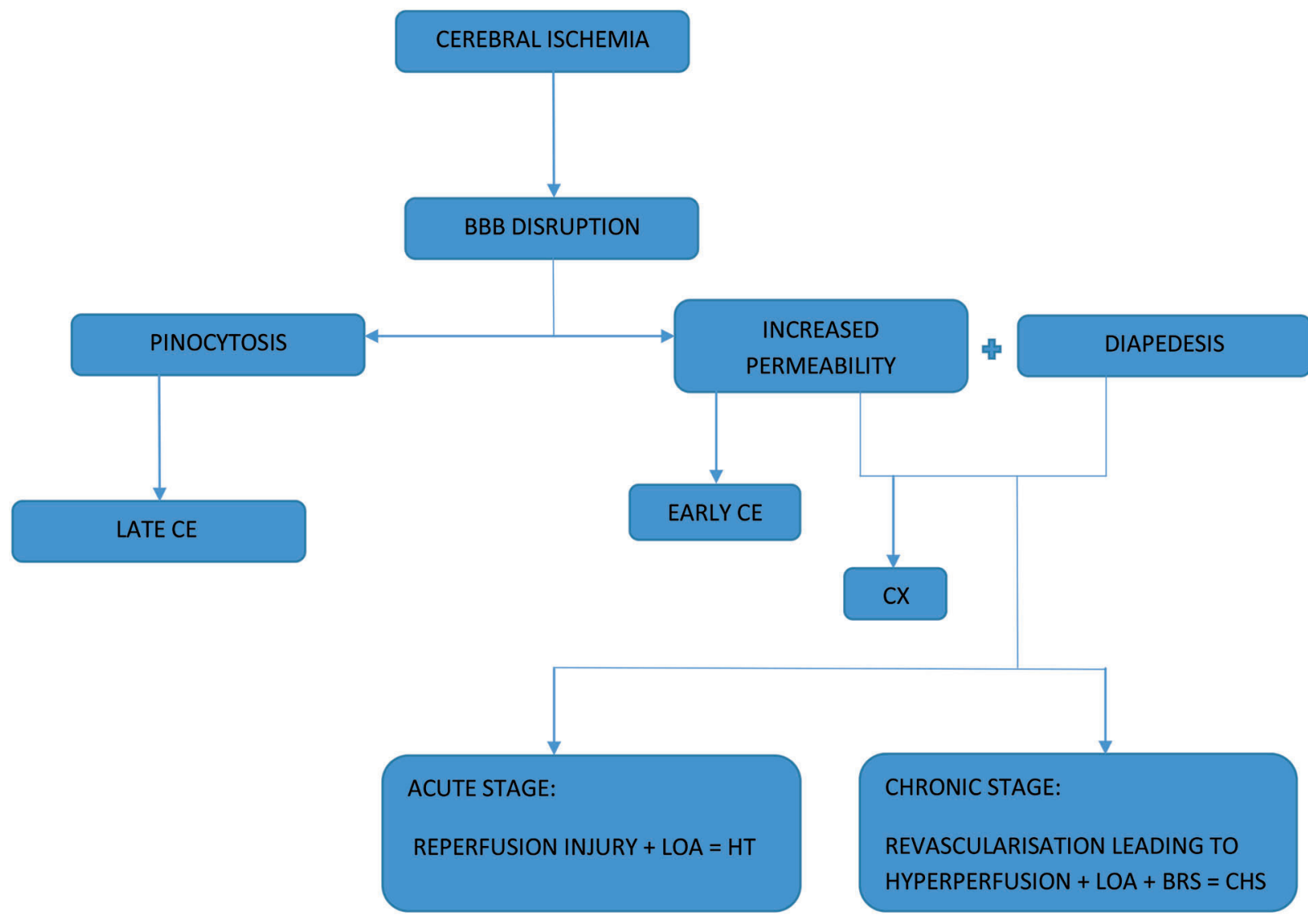

Fig. 2 Flowchart showing proposed mechanisms implicated in BBB disruption. BBB, blood brain barrier; BRS, baro recepter stimulation; CE, contrast enhancement; CHS, cerebral hyperperfusion syndrome; CX, contrast extravasation; HT, hemorrhagic transformation; LOA, loss of autoregulation.

\section{Contrast Enhancement and Contrast Extravasation}

CE versus $C X$ is a frequently faced dilemma on brain imaging of patients who had undergone ischemic revascularization procedure, and its sequel of hemorrhagic transformation is always feared. Solving this puzzle has a bearing on the future clinical course and recovery of the patients. We always hope for the former in immediate postthrombolysis CT scan. No one can surely bet in favor of the former or latter, and it takes 24 long hours to decide on follow-up imaging to know which way it is going. Disturbances in BBB are mainly responsible for letting either contrast media or blood cells to pass into extracellular spaces, resulting in either CE, CX, HT, or CHS.

\section{Contrast Enhancement (CE)}

Cerebral ischemia induces transmission of contrast through $B B B$, leading to contrast enhancement of infarcted region of the brain. Generally, it is seen in the first to fourth week. But it can be seen earliest on first day or a few months later after the ischemic insult. ${ }^{2}$ Various patterns of contrast enhancement seen on CT scan or MRI are: 1-gyral enhancement: gyral pattern of enhancement of cerebral cortex, 2-ring enhancement: ring of hyperdensity around an infarcted area, 3-patchy enhancement: with several small hyperdense areas diffusely scattered over the hypodense infarct, 4-homogenous enhancement: uniformly distributed within the infarcted region. ${ }^{3}$
Early stage of infarct associated with loosening of tight junctions between endothelial cells of the BBB result in increased permeability. This allows only contrast to go out and not the blood cells, thus resulting in contrast staining of the tissues. It depends on speed and extent of infarct. Faster and extensive infarcts are associated with early CE. Gyral pattern and homogenous pattern are more commonly seen in early stage and early recanalization, which is associated with intravenous thrombolysis. Late enhancement is attributed to impairment of BBB, which is augmented by pinocytosis and neovascularization. It does not depend on severity of infarction. As a definition, the contrast enhancement should disappear in 24 hours without leaving behind a residual cavity or mass effect. Both early and late CE are reversible. Late enhancement is part of healing stage and followed by replacement of brain volume, resulting in encephalomalacia. Early CE is less commonly associated with hemorrhagic transformation than contrast enhancement $(\boldsymbol{- F i g}$. 4)

Illustrative case 1 (CE): A 47-year-old housewife, known case of hypertension and diabetes, presented in January 2010 with right hemiparesis beyond 24 hours of onset. The National Institutes of Health Stroke Scale (NIHSS) score was 14 at admission. She showed left middle cerebral artery (MCA) and its watershed territory with PCA infarction. She recovered significantly with modified Rankin scale (MRS) score of 2 after 1 week. Digital subtraction angiography (DSA) at 


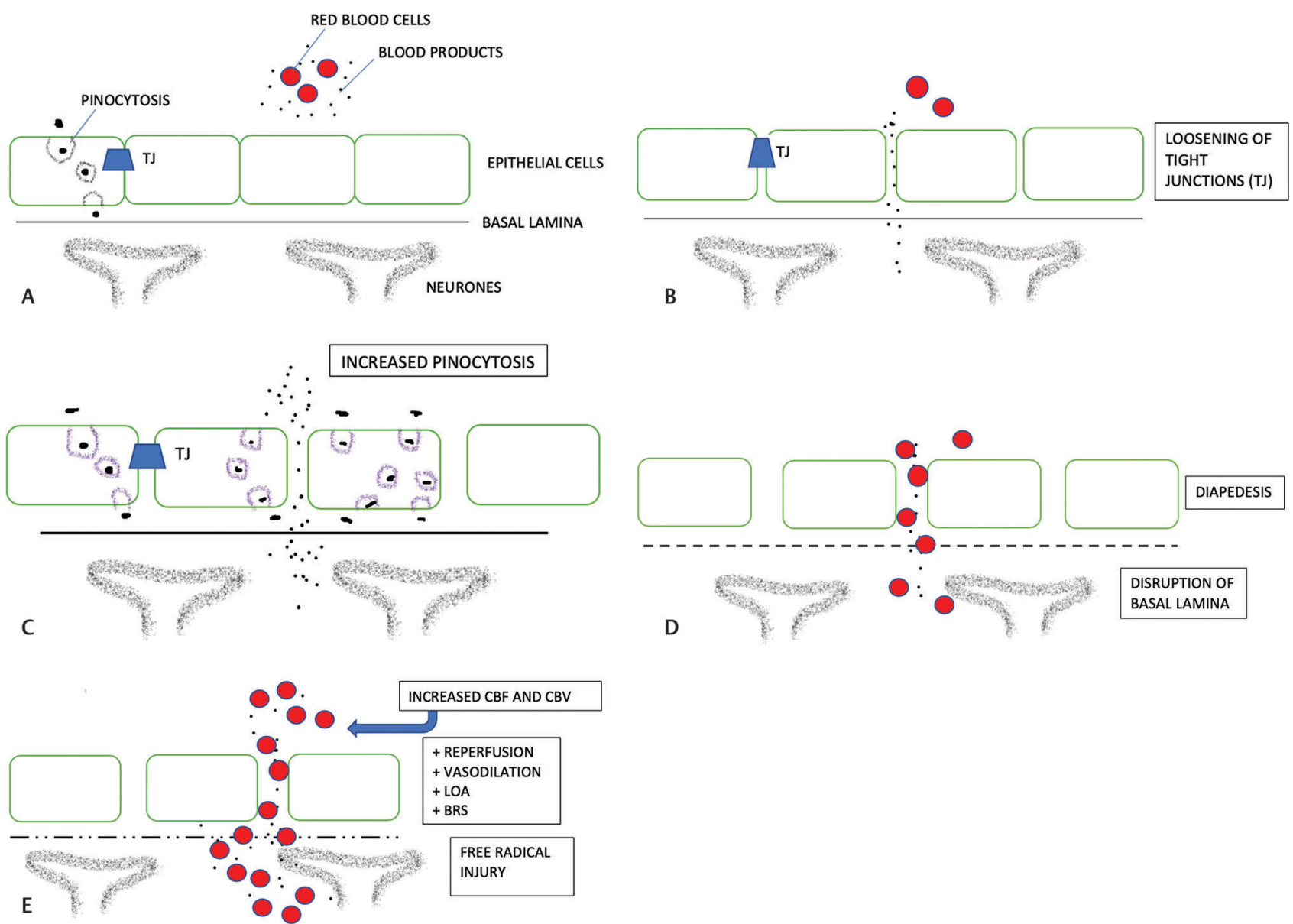

Fig. 3 Normal structure of blood-brain barrier (BBB) (A), mechanism of early contrast enhancement (CE) (B), mechanism of late CE (C), mechanism of contrast extravasation (CX) (D), mechanism of hemorrhagic transformation (HT) and cerebral hyperperfusion syndrome (CHS) (E).

this point showed left MCA significant stenosis. Intracranial stenting with coronary stent (Resolute, Medtronics, Dublin, Ireland) followed by balloon angioplasty (Maverik balloon catheter (Boston,Scientific) were done under general anesthesia (GA). Postextubation, she did not show any new neurological deficit. CT scan showed significant contrast stain, which washed out completely within 24 hours, suggesting it is CE. She had a MRS score of 1 at 3 months follow-up ( - Fig. 4 ).

\section{Contrast Extravasation (CX)}

$\mathrm{CX}$, on the other hand, persists longer than $\mathrm{CE}$ after the ischemic insult. It is defined as a hyperdense lesion with maximum Hounsfield unit $>90$ which persists beyond 24 hours on a follow-up CT scan. The basal lamina is a structural barrier preventing the cellular blood elements from extravasating from microvessels. The degradation of basal lamina along tissue necrosis leads to diapedesis. The more extensive infarcts result in more widespread necrosis and diapedesis. It may be postulated that $\mathrm{CX}$ is associated with parenchymal hemorrhage due to loss of structural integrity of the basal lamina. The infarcts which show CX have positive predictive value for hemorrhagic transformation. ${ }^{4}$

Post intra-arterial thrombolysis CT scan showing CE has positive prognostic value than those showing CX. Hence, management of hypertension and antithrombotic treatment regime should be guided accordingly to prevent hemorrhagic transformation. ${ }^{5}$ It is observed that contrast media itself may be adding to the BBB damage and neurotoxicity Hence, it is advisable to minimize the contrast injections during intra-arterial thrombolytic therapy. 4,5 Accordingly, contrast injections through microcatheter should be avoided, as they are known to be associated with increased hemorrhagic risk. ${ }^{6}$ The thrombolytic agents also have deleterious effects on BBB; hence, its judicious use is recommended ( - Fig. 5).

Illustrative case 2 (CX): A 52-year-old female teacher, known case of hypertension, presented in June 2009 with sudden onset left hemiplegia. CT scan showed no infarct with right MCA dense sign. She was thrombolyzed with recombinant tissue thromboplastin intravenously outside our facility. On admission, NIHSS score was 10 . Time from onset was 3 hours. DSA showed right terminal internal carotid artery (ICA) occlusion, which was recanalized by manual aspiration and coronary balloon angioplasty (Maverik balloon catheter, Boston scientific, Massachusetts, USA). However, immediate postmechanical thrombolysis CT showed contrast opacification, which was partially cleared in 24 hours, suggesting CX. She further deteriorated within the next 1 week with large infarct of right MCA territory, which required decompressive craniectomy. She showed gradual recovery with a MRS score of 3 at 3 months follow-up (-Fig. 5). 

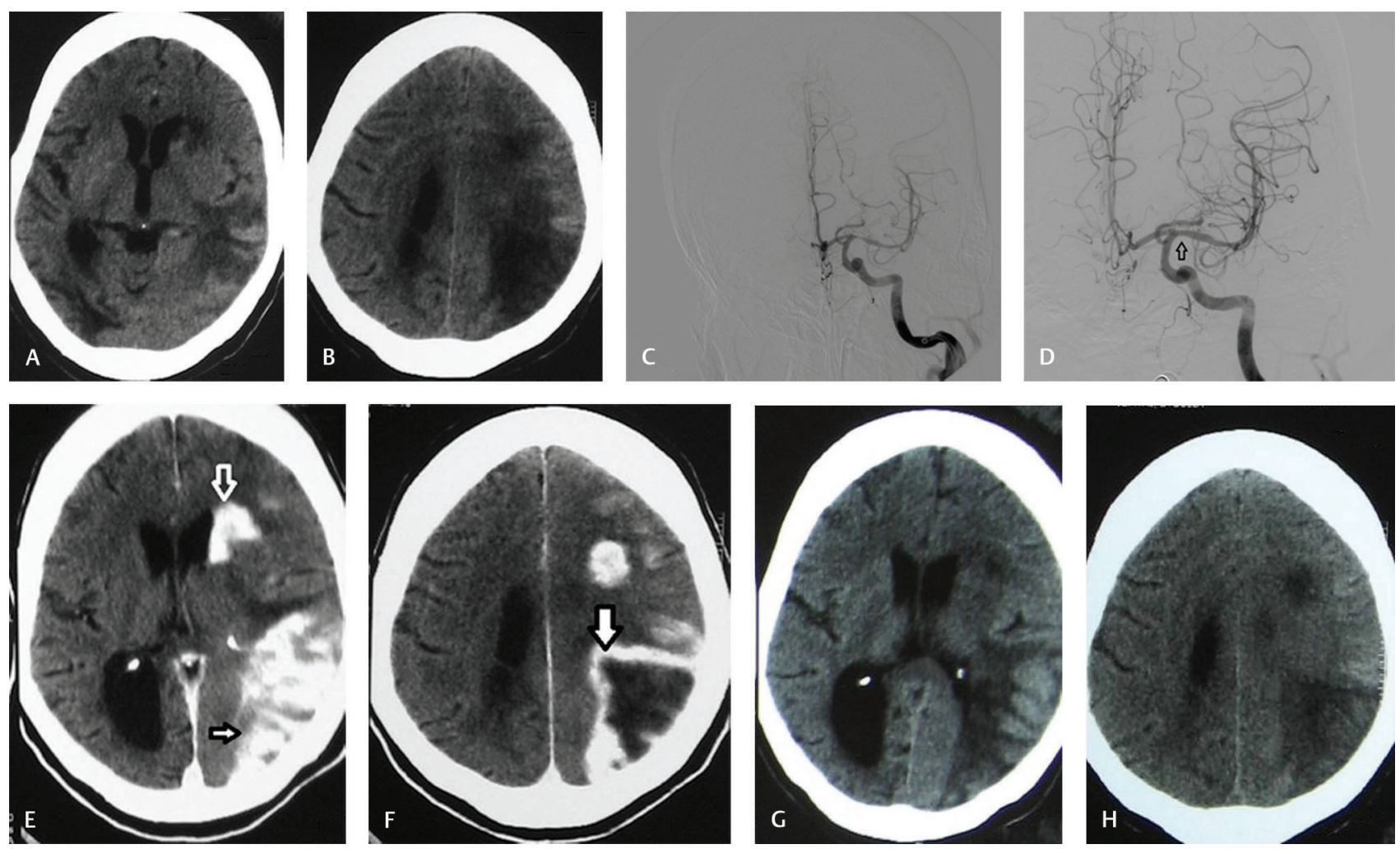

Fig. 4 Axial CT scan (A,B) showed middle cerebral artery (MCA) territory infarction. Digital subtraction angiography (DSA) images pre (C) and post (D) MCA stenting. Axial CT scan images immediately after the stenting (E,F) showed contrast staining with homogenous pattern (arrow: white outline), gyral pattern (arrow: black outline), and peripheral rim pattern (arrow: solid white with black outline), which cleared which 24 hours, as seen in axial CT scan $(\mathbf{G}, \mathbf{H})$, confirming it was contrast enhancement.
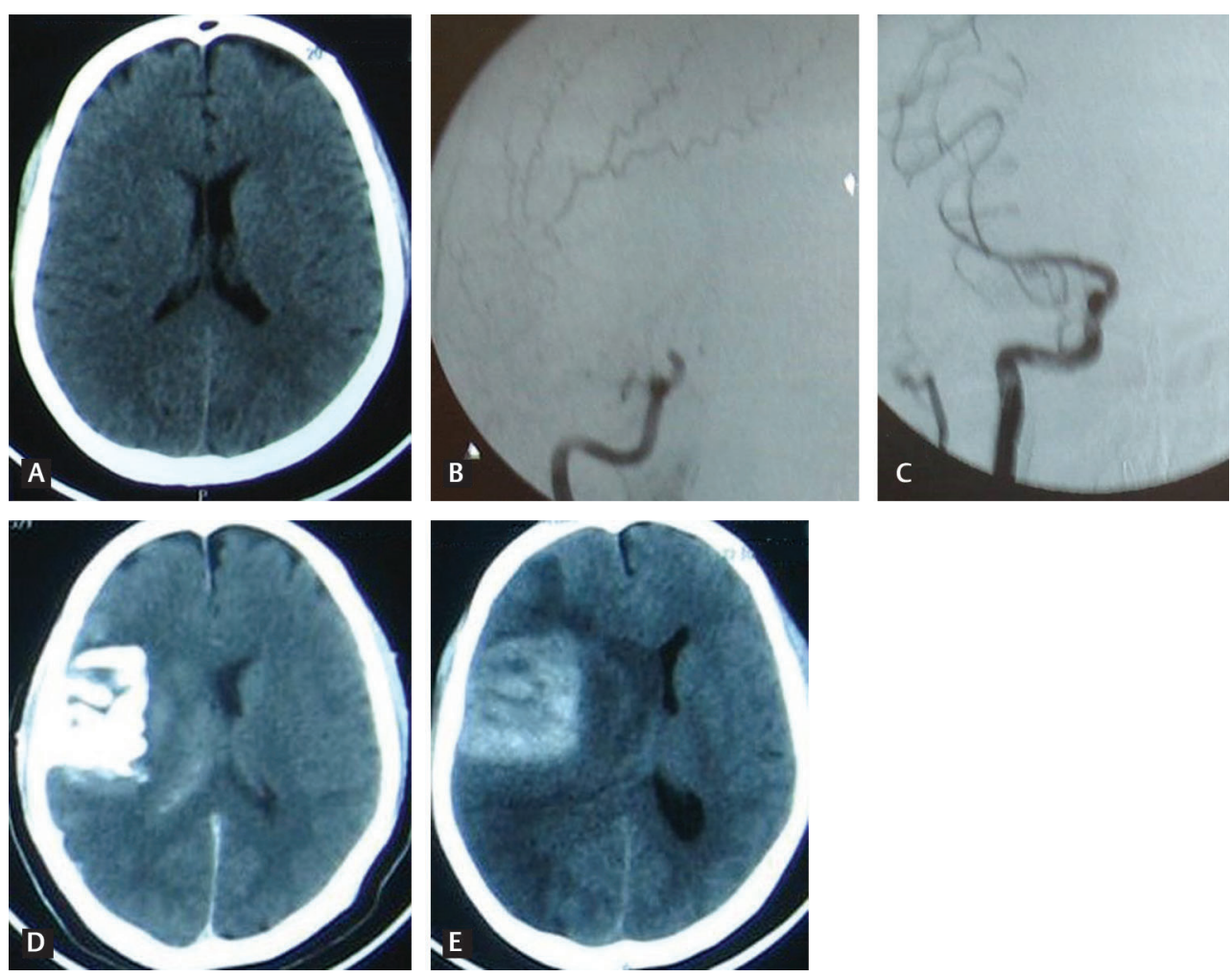

Fig. 5 Axial CT scan (A) showed no infarction. Digital subtraction angiography (DSA) image (B) showed right terminal internal carotid artery (ICA) occlusion. Postthrombolysis DSA image (C) showed complete recanalization. Immediate postthrombolysis axial CT scan (D) showed gyral contrast staining, which persisted beyond 24 hours, as seen in image (E). Eventually this patient developed large middle cerebral artery (MCA) infarction due to reocclusion (not shown here). Imaging findings confirm this is a case of contrast extravasation (CX). 


\section{Hemorrhagic Transformation (HT)}

Hemorrhage and infarction are a lethal combination. Hemorrhagic transformation of bland infarct is a complex and dynamic biochemical event related to ischemic neuronal damage. Ischemia initiates a cascade involving reduction in oxygen and glucose supply, turning into less ATP production and thereby resulting in defunct sodium potassium pumps. This leads to cellular and metabolic disharmony, creating disruptions in the BBB neuronal necrosis along with cytokine and free nitric oxide species inflicting diapedesis which, in turn, leads to hemorrhage. Reperfusion, also called luxury perfusion, may be due spontaneous dissolution of thrombus or as result of acute thrombolytic therapies. Reperfusion is desirable to salvage brain tissues, but it has deleterious effects too. Free oxygen radical injury and inflammatory responses add insult to injury with more disruptions of BBB. All these events causing hyperemia after the neuronal death are detrimental to BBB integrity, opening gates for cellular components of blood into infracted field. ${ }^{7,8}$ It is proved that reopened vessels in the infarcted tissues increases chances of HT. Disruption of BBB coupled with increased cerebral blood volume (CBV) caused by reperfusion and vasodilation due to loss of cerebrovascular autoregulation increases likelihood of hemorrhagic transformation. Hemorrhagic transformation is divided into hemorrhagic infarction (HI) and parenchymal hematoma (PH). Radiologically, these two types and their subtypes are distinguishable. HI refers to the pathological condition in which petechial or more confluent hemorrhages occupy a portion of an area of ischemic infarction. PH in an area of infarction containing solid clot of blood with mass effect. ${ }^{6} \mathrm{HI}$ is further divided into $\mathrm{HI}-1$ and $\mathrm{HI}-2$. $\mathrm{HI}-1$ is as small petechiae along the margins of the infarct, and $\mathrm{HI}-2$ is petechiae within all the infarcted area, but without space-occupying effect. $\mathrm{PH}-1$ is defined as a clot not exceeding $30 \%$ of the infarcted area with some mild space-occupying effect. $\mathrm{PH}-2$ represents dense blood clot(s) exceeding 30\% of the infarct volume with significant space-occupying effect. ${ }^{9-12}$ This stratification is clinically significant, and $\mathrm{PH}-2$ is associated with increased morbidity and mortality. Intravenous thrombolytic agents increase the risk of hemorrhage along with risk of early hemorrhagic transformation. Late time of Initiation, high NIHSS, low Alberta stroke programme early CT score (ASPECTS), smokers, increased activated partial thromboplastin time, low platelet count, and low fibrinogen levels are independent predictors of HT. ${ }^{4,13-16}$ Even mechanical thrombolysis is associated with HT in around $10 \%$ of cases. Old age, large infarct core, GA, and unfavorable collaterals are additional risk factors for $\mathrm{HT}^{17}$ (-Fig. 6).

Illustrative case 3 (CX with HT): A 21-year-old employee smoker, known case of nonhypertension and nondiabetic status, presented in February 2020 with sudden onset right hemiplegia along with aphasia within 2 hours of onset. The NIHSS score was 16 at admission. CT scan showed no significant infarct. In view of clinical finding suggesting large vessel occlusion, he was directly taken for mechanical thrombolysis. Complete recanalization with thrombolysis in myocardial infarction (TIMI)-3 flow was achieved in 20 minutes of grain to reperfusion time by penumbra aspiration system (Penumbra, California, USA). Immediate postthrombolysis CT scan showed contrast staining, which persisted beyond
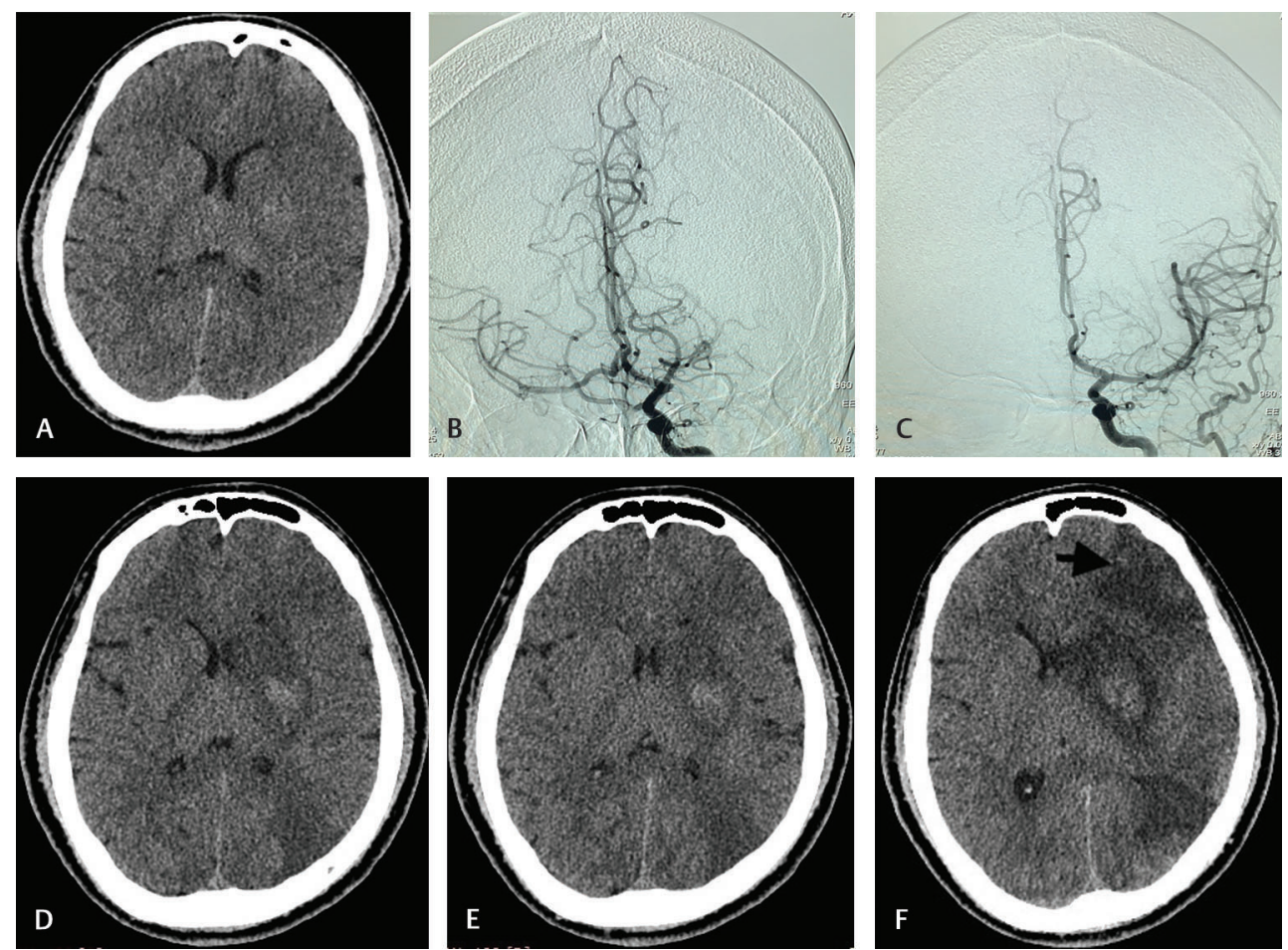

Fig. 6 Axial CT scan prior to thrombolysis does not show infarction or hemorrhage (A). Digital subtraction angiography (DSA) showed middle cerebral artery (MCA) occlusion (B), which was recanalized by mechanical thrombolysis (C). Immediate postthrombolysis CT scan showed contrast staining (D), which persisted beyond 24 hours (E), and after 1 week, it showed increase in size of infarctions with petechial hemorrhages (F) marked by arrow: solid black. The imaging findings confirmed this as case of contrast extravasation (CX) with later hemorrhagic transformation. 
24 hours and was suggestive of CX. He developed few petechial hemorrhages after 1 week. He recovered with MRS score of 2 at 3 months follow-up (-Fig. 6).

\section{Cerebral Hyperperfusion Syndrome (CHS)}

It is defined as focal or global neurological dysfunction, leading to death due to hyperperfusion following revascularization. It is rare, potentially fatal, but a possibly preventable event associated with revascularization like CEA and CAS. According to Bouri et al, four criteria to define CHS should be present: 1-Occurrence within 30 days of revascularization, 2-Clinical features such as new onset headache, seizure, hemiparesis, GCS score $<15$, or radiological features including cerebral edema and intracerebral hemorrhage (ICH), 3-Evidence of hyperperfusion (on transcranial Doppler [TCD], single-photon emission computed tomography [SPECT] or CT/MR perfusion imaging) or systolic blood pressure (SBP) > $180 \mathrm{~mm} \mathrm{Hg}$, 4No evidence of new cerebral ischemia, postoperative carotid occlusion, and metabolic or pharmacologic cause. ${ }^{18-20}$

Pathophysiological mechanisms involved in CHS are increased perfusion superadded with BBB disruption, which is associated with ischemia or infarction, impairment of cerebrovascular autoregulation, baroreceptor denervation or stimulation, chronic hypertension, microangiopathy, and free radical injury. ${ }^{20}$ Predisposing factors associated with CHS are high-grade stenosis, contralateral occlusion, and bilateral high-grade stenosis. The imaging modalities used to diagnose CHS are TCD, CT scan, MRI, MR perfusion studies, and SPECT scan. Determination of hyperferfusion and loss of cerebral vascular reserve which is an ominous sign of CHS prior to revascularization is advisable. Preventive measures are suggested, such as controlling blood pressure in the postoperative period in the range of 140 to $160 \mathrm{~mm}$ of $\mathrm{Hg}$, targeting the preprocedure if it is lower than that, and watching for clinical symptoms of headache, seizures or neurodeficit. Early stage of CHS with cerebral edema is reversible and can be detected on follow-up CT scan whenever in doubt. It should be meticulously managed, and every effort should be made to avoid hemorrhagic stage. Antihypertensives with mixed $\alpha$ and beta blockers like labetolol are preferred, as they have no significant effect on intracranial pressure, and they do not hamper autoregulation. These medications decrease cerebral perfusion pressure and mean arterial pressure without altering CBF. Antihypertensives which have vasodilator effect, like calcium channel blockers, sodium nitroprusside, glycerol trinitrate, and angiotensin II inhibitors, should be avoided. Antiedema measures with mannitol and hypertonic saline along with free radical scavengers will be helpful. It is generally accepted that its incidence is more in CEA than CAS. The systematic reviews suggest CHS after CAS to be up to $1.1 \%$ (-Fig. 7 ).

Illustrative case 4(CHS): A 67-year-old farmer smoker with history of hypertension and diabetes since the last 20 years.
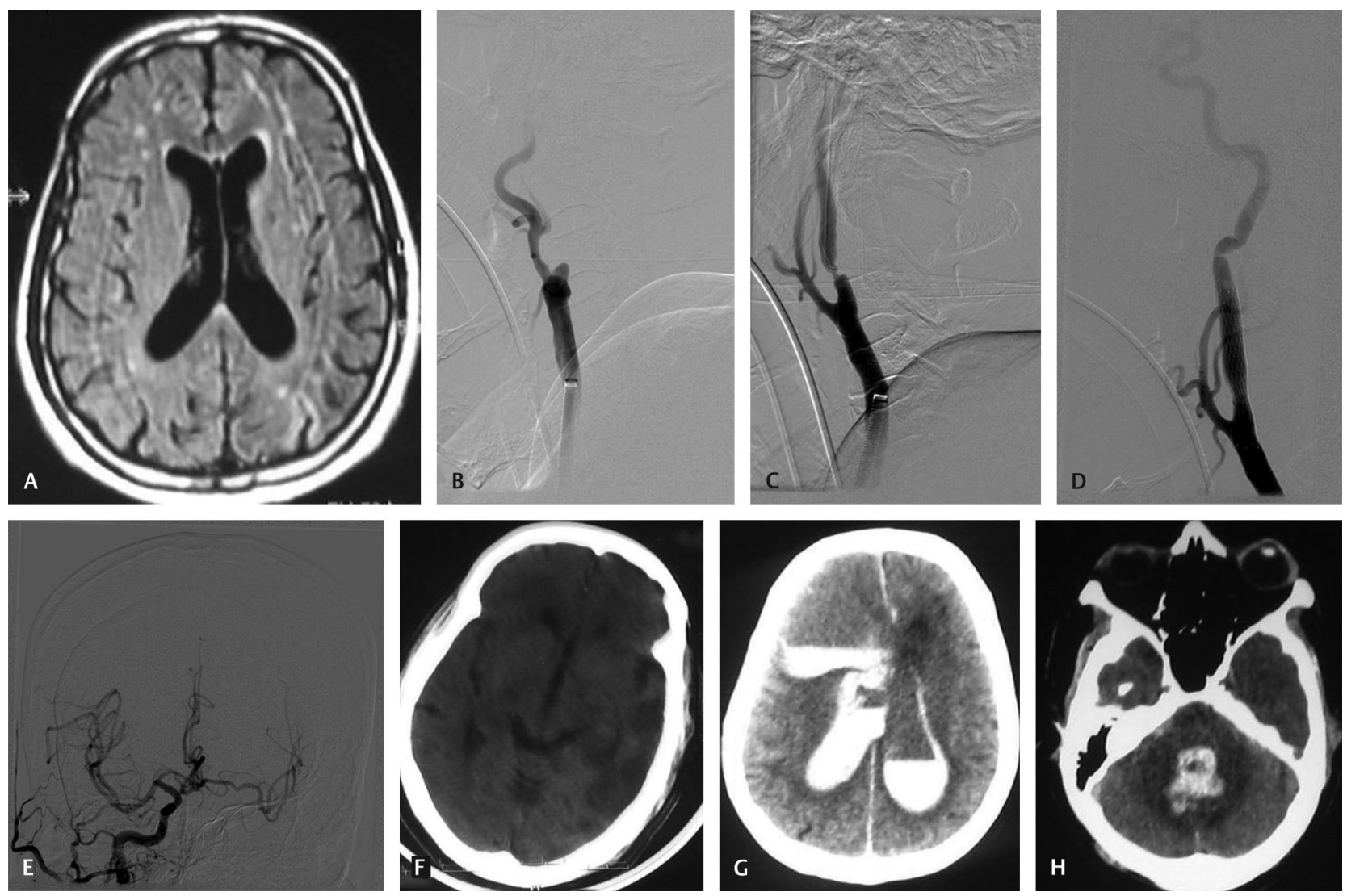

Fig. 7 Pre-carotid stenting (CAS) MRI showed white matter microangiopathic changes (A). Digital subtraction angiography (DSA) showed left internal carotid artery (ICA) occlusion (B) and right ICA $80 \%$ stenosis (C) for which CAS was done, as shown in image (D). Intracranial circulation with cross-flow across anterior communication (ACOM) artery. (E). Immediate post CAS CT scan (F) does not show any new infarction or hemorrhage. CT after 30 minutes showed intracranial hemorrhage with intraventricular extension (G, $\mathbf{H})$. Findings confirmed this is case of cerebral hyperperfusion syndrome (CHS) with intracranial hemorrhage. 
He presented in May 2009 with left-sided weakness over the last 48 hours. The NIHSS score was 2 at the time of admission. He had left-sided sudden loss of vision 2 years back, which recovered in 20 minutes for which he was started on single antiplatelet (ecosprin $150 \mathrm{mg}$ daily). MRI scan showed multiple white matter and periventricular punctuate lesions (Fazekas grade 1). DSA showed complete occlusion of left ICA at carotid bulb. Right ICA showed $80 \%$ stenosis by way of the North American symptomatic carotid endarterectomy trial (NASCET) method. Blood sugar was controlled. He was started on double antiplatelets with Clopidogrel 75 along with Ecosprin $150 \mathrm{mg}$ daily. He was on angiotensin-converting enzyme (ACE) inhibitor for blood pressure and metformin $500 \mathrm{mg}$ for diabetes. Due to contralateral occlusion, we decided to do right carotid stenting under GA. His preprocedure blood pressure was 138/84 mm of Hg. Carotid stenting was uneventful. Immediately after the procedure, on extubation, his blood pressure was 140/84. After 15 minutes, he started complaining severe headaches with blood pressure 180/110. There was no new neurological deficit. CT scan at this point did not show any new infarct or hemorrhage. Labetelol infusion started; 5 minutes later, he had generalized tonic clonic seizure followed by loss of unconsciousness. He registered a GCS score of 6/15. CT scan at this point showed right intracranial hemorrhage with intraventricular extension suggesting CHS. He expired in the next 30 minutes (-Fig. 7).

\section{Conclusion}

BBB disruption is the primary event in CE, CX, HT, and CHS with varying severity. Imaging can diagnose all these with classical features as described above. CT scan is sensitive to detect CE, CX, and HT. However, MRI can pick up more minute HT changes with gradient echo and susceptibility sequences, but clinical implication of MRI-based changes are not yet validated. Edema stage of CHS will be better determined on MRI than $\mathrm{CT}$ but hyperperfusion and cerebrovascular reserve will be exclusively seen on TCD and SPECT scan. Minimizing dose of contrast, optimum timing of revascularization and dose of thrombolytic, judicious selection of mechanical thrombectomy cases, and strict control of blood pressure in post revascularization period are recommended preventive measures. High-index of clinical suspicion, early imaging to detect, and following-up the same on sequential imaging are key to avoiding severe forms of HT and CHS.

\section{Conflicts of Interest}

None declared.

\section{References}

1 Smirniotopoulos JG, Murphy FM, Rushing EJ, Rees JH, Schroeder JW. Patterns of contrast enhancement in the brain and meninges. Radiographics 2007;27(2):525-551

2 Norton GA, Kishore PR, Lin J. CT contrast enhancement in cerebral infarction. AJR Am J Roentgenol 1978;131(5):881-885
3 Hornig CR, Busse O, Buettner T, Dorndorf W, Agnoli A, Akengin Z. CT contrast enhancement on brain scans and blood-CSF barrier disturbances in cerebral ischemic infarction. Stroke 1985;16(2):268-273

4 Yoon W, Seo JJ, Kim JK, Cho KH, Park JG, Kang HK. Contrast enhancement and contrast extravasation on computed tomography after intra-arterial thrombolysis in patients with acute ischemic stroke. Stroke 2004;35(4):876-881

5 Merten CL, Knitelius HO, Assheuer J, Bergmann-Kurz B, Hedde JP, Bewermeyer H. MRI of acute cerebral infarcts, increased contrast enhancement with continuous infusion of gadolinium. Neuroradiology 1999;41(4):242-248

6 Khatri R, Khatri P, Khoury J, Broderick J, Carrozzella J, Tomsick T. Microcatheter contrast injections during intra-arterial thrombolysis increase intracranial hemorrhage risk. J Neurointerv Surg 2010;2(2):115-119

7 Fiorelli M, Bastianello S, von Kummer R, et al. Hemorrhagic transformation within 36 hours of a cerebral infarct: relationships with early clinical deterioration and 3-month outcome in the European Cooperative Acute Stroke Study I (ECASS I) cohort. Stroke 1999;30(11):2280-2284

8 Zhang J, Yang Y, Sun H, Xing Y. Hemorrhagic transformation after cerebral infarction: current concepts and challenges. Ann Transl Med 2014;2(8):81

9 Pessin MS, Del Zoppo GJ, Estol CJ. Thrombolytic agents in the treatment of stroke. Clin Neuropharmacol 1990;13(4):271-289

10 del Zoppo GJ, Poeck K, Pessin MS, et al. Recombinant tissue plasminogen activator in acute thrombotic and embolic stroke. Ann Neurol 1992;32(1):78-86

11 Hacke W, Kaste M, Fieschi C, et al; The European Cooperative Acute Stroke Study (ECASS). Intravenous thrombolysis with recombinant tissue plasminogen activator for acute hemispheric stroke. JAMA 1995;274(13):1017-1025

12 von Kummer R, Broderick JP, Campbell BC, et al. The Heidelberg Bleeding Classification: classification of bleeding events after ischemic stroke and reperfusion therapy. Stroke 2015;46(10):2981-2986

13 Wang R, Zeng J, Wang F, Zhuang X, Chen X, Miao J. Risk factors of hemorrhagic transformation after intravenous thrombolysis with rt-PA in acute cerebral infarction. QJM 2019;112(5):323-326

14 National Institute of Neurological Disorders and Stroke rt-PA Stroke Study Group. Tissue plasminogen activator for acute ischemic stroke. N Engl J Med 1995;333(24):1581-1587

15 Hacke W, Kaste M, Fieschi C, et al; Second EuropeanAustralasian Acute Stroke Study Investigators. Randomised double-blind placebo-controlled trial of thrombolytic therapy with intravenous alteplase in acute ischaemic stroke (ECASS II) Lancet 1998;352(9136) :1245-1251

16 Hacke W, Kaste M, Bluhmki E, et al; ECASS Investigators. Thrombolysis with alteplase 3 to 4.5 hours after acute ischemic stroke. N Engl J Med 2008;359(13):1317-1329

17 Boisseau W, Fahed R, Lapergue B, et al; ETIS Investigators. Predictors of parenchymal hematoma after mechanical thrombectomy: a multicenter study. Stroke 2019;50(9):2364-2370

18 Bouri S, Thapar A, Shalhoub J, et al. Hypertension and the post-carotid endarterectomy cerebral hyperperfusion syndrome. Eur J Vasc Endovasc Surg 2011;41(2):229-237

19 Farooq MU, Goshgarian C, Min J, Gorelick PB. Pathophysiology and management of reperfusion injury and hyperperfusion syndrome after carotid endarterectomy and carotid artery stenting. Exp Transl Stroke Med 2016;8(1):7

20 Galyfos G, Sianou A, Filis K. Cerebral hyperperfusion syndrome and intracranial hemorrhage after carotid endarterectomy or carotid stenting: A meta-analysis. J Neurol Sci 2017;381:74-82 